\title{
Kommentar zum Supply Chain Event Management
}

\author{
Anmerkungen zum Beitrag \\ Tröger, Ralph und Alt, Rainer: Serviceorientiertes Supply Chain Event \\ Management (SCEM) - Nutzen und Architektur für globale Lieferketten am \\ Beispiel der Modeindustrie
}

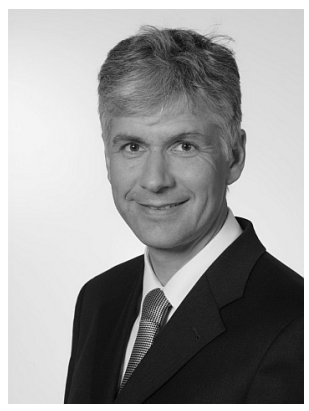

\section{Wolfgang Stölzle}

\section{Relevanz}

Als eine der grossen Barrieren für die Umsetzung von Supply Chain Management in der Unternehmenspraxis gilt die grosse Komplexität von Wertschöpfungsnetzwerken. Die sich mit zunehmender Arbeitsteiligkeit reduzierenden Wertschöpfungstiefen pro Akteur erhöhen die Zahl der Supply Chain-Mitglieder. Die Globalisierung führt zu einer stärkeren Dislozierung der Wertschöpfungsstandorte und damit zu grösseren Transportdistanzen in den Netzwerken. Dies steigert insgesamt den Koordinationsaufwand in globalen Supply Chains erheblich und erfordert längere Zeitfenster für die operativen Abstimmungen zwischen den Supply ChainMitgliedern. Der Trend bei der Ausgestaltung von Supply Chains geht jedoch - den Paradigmen von „lean“ und „agile“ folgend - in Richtung klein dimensionierter Pufferbestände, die in Anbetracht der starken Marktdynamik und hohen Empfindlichkeit kurze Reaktionszeiten und schnelle Anpassungsentscheidungen erfordern. Damit bildet sich eine Zeitschere heraus: Die höhere Komplexität von Wertschöpfungsnetzwerken erfordert zeitaufwändige Koordinations- und Entscheidungsprozesse, die Ausgestaltung der Lieferketten zeichnet sich durch eine hohe Empfindlichkeit aus und verlangt kurze Reaktionszeiten. Der Handlungsbedarf für die Entwicklung von Supply Chain Event Management (SCEM) lässt sich aus dem Schliessen dieser Zeitschere ableiten.

Die Ursprünge von SCEM liegen im Tracking \& Tracing (T\&T) begründet, das bereits in den 80er Jahren in hoch-industrialisierten Logistik- und Transportnetzwerken der Kurier-, Paket- und Expressbranche Einzug gehalten hat. Gegenüber T\&T-Lösungen, die Statusinformationen von Sendungen dokumentieren, ist SCEM mit deutlich mehr Intelligenz versehen. Statt den Nutzer ungerichtet mit einer grossen Zahl von Statusinformationen von Sendungen zu versorgen und ihn damit auch mit einer grossen Datenmenge zu belasten, verfolgt SCEM den Anspruch, das Management sehr gezielt zu unterstützen. Solange Supply Chain-Prozesse vorab definierte Standards einhalten, sollen die Entscheidungsträger möglichst nicht mit dann für sie meist unbedeutenden Informationen „belastet“ werden. Sobald jedoch bei einer Prozessausführung vorgegebene Toleranzwerte ausgewählter Prozessparameter überschritten werden, sind die Entscheidungsträger zu informieren. Hierbei setzen intelligente Eskalationsmechanismen ein: Für bestimmte Abweichungen sind spezifische Handlungsimpulse vorgedacht. Je nach Bedeutung einer Abweichung von 
den Prozess-Standards werden zudem Entscheidungsträger bestimmter Hierarchieebenen adressiert. Damit bilden ,Events‘ den Schlüsselbegriff des Konzepts: Sie kennzeichnen das Überschreiten der Toleranzgrenzen von Prozessparametern in Supply Chains und nehmen den Charakter kritischer Ausnahmeereignisse ein.

Die Mächtigkeit einer globalen Supply Chain kommt über eine Vielzahl eingebundener Akteure mit jeweils eigenen Supply Chain-Prozessen, einem Artikelspektrum im oft 6-stelligen Bereich und häufig mehreren Tausend Sendungen pro Tag zum Ausdruck. Damit ist das SCEM mit einem beachtlich grossen Datenvolumen konfrontiert, das nur mit leistungsfähigen IT-Lösungen im Hinblick auf die Identifikation von Events und die Unterstützung der Eskalationsmechanismen bewältigt werden kann. Deshalb wird eine starke Serviceorientierung der IT-Lösungen für deren Anwender unabdingbar. Insofern kann dem Beitrag von Ralph Tröger und Rainer Alt zum Service-orientierten SCEM eine hohe Relevanz attestiert werden.

\section{Argumentation}

Die Auseinandersetzung mit dem SCEM-Konzept in Wissenschaft und Praxis während der letzten Jahre zeigt, dass der Konzeptumsetzung deutlich zu wenig Aufmerksamkeit geschenkt wurde. Insofern machen die Autoren des Beitrags ihre Argumentation plausibel an den Nutzenkomponenten von SCEM fest. Die Nutzenpotenziale von SCEM werden nach Massgabe der beiden Dimensionen Nutzenebene (operativ/taktisch/strategisch) und Nutzenkategorie (quantitativ/qualitativ) unterteilt. Allerdings wählen sie für die anschliessende Analyse der Nutzeneffekte von Service-orientierten Architekturen (SOA) der IT-Lösungen für SCEM eine abweichende Systematisierung in eine Kosten-, Zeit- und eine Qualitätsebene. Dies erleichtert nicht unbedingt die Stringenz der Argumentation im Hinblick auf eine Verknüpfung von Service-orientierten Architekturen (SOA) mit dem SCEM-Konzept. Auch der nicht präzisierte Begriff des „Nutzentreibers“ in der Überschrift von Kapitel 2 hilft hier nicht unbedingt weiter.

Die Kernleistung der Autoren in Gestalt der Ableitung von Nutzenpotenzialen des SCEM unter Einbeziehung von SOA setzt offenbar auf der Entwicklung eines Frameworks zur Identifizierung von SCEM-Services (siehe Abbildung 3 im Beitrag von Tröger/Alt) auf, der in eine Systemarchitektur für ein Service-orientiertes SCEM überführt wird (siehe $A b$ bildung 4 im Beitrag von Tröger/Alt). Hierbei transferieren die Verfasser ihre Strukturierungsleistung auf eine Fallstudie in der Modeindustrie und integrieren damit einen Anwendungsbezug in ihre Ausführungen.

\section{Kritische Würdigung}

Insgesamt gefällt die anschauliche Illustration eines relevanten Themenfeldes ebenso wie die konzeptionelle Idee für eine geeignete Weiterentwicklung des SCEM, insbesondere mit Blick auf dessen Umsetzung in der Supply Chain-Praxis.

Die Aussage der Autoren, mit ihrer Erkenntnis einen Beitrag zur der Generierung von Wettbewerbsvorteilen geleistet zu haben, steht allerdings etwas offen im Raum: Denn es erfolgt beispielsweise keine Analyse, inwieweit die Kunden einer Supply Chain die Nutzeneffekte eines Service-orientierten SCEM in Gestalt zusätzlicher Zahlungsbereitschaft honorieren. In Anbetracht der z.T. erheblichen Investitionen in SCEM-Lösungen wäre unter Berücksichtigung etwaiger zusätzlicher Zahlungsbereitschaft zu prüfen, wie sich eine solche 
Systemarchitektur auf die Wirtschaftlichkeit der Supply Chain bzw. deren Performance auswirkt.

Weiterhin gilt die Akzeptanz aus Sicht der Anwender, d. h. der Supply Chain-Akteure, bisher als grosse Barriere für das SCEM. Auch dieser Punkt wird von den Autoren nicht adressiert, obwohl sie Praxisrelevanz für sich reklamieren. Beispielsweise wäre zu diskutieren, welche organisatorischen Voraussetzungen bei den Supply Chain-Akteuren für ein Service-orientiertes SCEM erfüllt sein müssen und wie die Akteure mit der verbesserten Supply Chain-Visibility bei der operativen Steuerung von Supply Chains umgehen.

Weiterhin werden mit Blick auf die Umsetzung des SCEM die Anforderung der unternehmensübergreifenden Prozess-Standardisierung und die notwendige Verknüpfung der IT-Lösungen der Supply Chain-Akteure nicht ausreichend beleuchtet. Dazu gehört auch die ausbleibende Auseinandersetzung mit der Robustheit des Service-orientierten SCEM gegenüber Modifikationen von Supply Chain-Prozessen. Bei spezifischen Supply ChainDesigns stellt sich zudem die Frage, inwieweit solche Service-orientierten Lösungen hochintegrierte Supply Chain-Konzepte abzubilden vermögen.

Schliesslich hätte eine tiefergehende Analyse einer konkreten beispielhaften Prozesskette der Modeindustrie mit Zielgrössen, Prozess-Standards und -Parametern, Toleranzgrenzen, der Definition von kritischen Ereignissen und einem Eskalationsmanagement die praktischen Implikationen verdeutlichen können. So bleibt für den Leser offen, inwiefern die Ausführungen zum Beispiel der Modeindustrie einerseits wirklich umsetzbar und andererseits generalisierbar sind.

Insgesamt bietet die Arbeit von Ralph Tröger und Rainer Alt eine interessante Idee zur verbesserten Umsetzbarkeit des SCEM-Konzepts. Viele Fragen bleiben jedoch noch offen, so dass weiteren Forschungsinitiativen nichts im Weg steht.

\section{Literaturhinweise}

Reiche, F., et al. (2009): Ereignisorientierte Steuerung von Lieferketten. Nutzen, aktueller Stand der Nutzung und Potenziale, Göttingen.

Wolfgang Stölzle, Prof. Dr., ist Ordinarius am Lehrstuhl für Logistikmanagement der Universität St.Gallen.

Anschrift: Universität St.Gallen, Lehrstuhl für Logistikmanagement, Dufourstrasse 40 a, CH-9000 St. Gallen, Tel.: +41 (0)71/224-7281, Fax: +41 (0)71/224-7315, E-Mail: wolfgang.stoelzle@unisg.ch 